\title{
Results and Lessons from the Operation of Current Beams for Existing Neutrino Experiments
}

\author{
Edda Gschwendtner ${ }^{1}$ \\ CERN \\ AB-Department, 1211 Geneva 23, Switzerland \\ E-mail: edda.gschwendtner@cern.ch
}

\begin{abstract}
An overview of the exploitation of accelerator neutrino beam facilities is presented. Emphasis is given on the elements of the secondary beam, i.e. the target and the equipment downstream of it. Using the examples of K2K, MiniBooNE, NuMI and CNGS, a number of lessons learnt in operating these neutrino beams are outlined. The T2K facility, presently under construction, is included in this overview.
\end{abstract}

10th International Workshop on Neutrino Factories, Super beams and Beta beams Valencia, Spain

30 June - 05 July, 2008

\footnotetext{
$1 \quad$ Speaker
} 


\section{Introduction}

The current beams for existing neutrino experiments are based on producing neutrinos from meson decay in flight. A proton beam is sent to a target, producing among many other particles pions and kaons. The charged $\pi / \mathrm{K}$ are energy-selected and guided using a magnetic focusing system. These mesons then decay in flight into muons and muon-neutrinos. Hadrons that have not interacted in the target or have not decayed are absorbed in a hadron stopper.

Physics requirements have been pushing the required power of the proton beam on target to values around and above $500 \mathrm{~kW}$. The design of the production target is a compromise between the probability of the protons to interact and the re-interaction or scattering of the particles produced. Moreover, beam impact and target heating is a major issue in the design, and some form of cooling is needed.

The focusing system is usually built using one or several magnetic 'horns' [1]. These cylindrical structures, producing a toroidal field, have a very thin inner conductor to minimize particle interactions. Pulsed currents exceeding $200 \mathrm{kA}$ are applied, requiring that the inner conductor be cooled - water-cooling is generally used.

The dimensions of the decay region depend on the energy of the mesons. Choosing to evacuate the decay tube or filling it with air/helium is weighed against the entrance window thickness and related safety issues.

The hadron absorber has to withstand the full beam power for at least one proton beam pulse (in case the beam misses the target) and has to continuously absorb some 5-10 percent of the beam power. All systems in operation and planned use water cooling of the hadron absorber.

Beam monitoring equipment is required upstream of the target to center the proton beam correctly. The radiation levels downstream of the target make it difficult to install sophisticated monitors. Beyond the hadron absorber, muon detectors provide valuable on-line information on the intensity and quality of the neutrino beam produced.

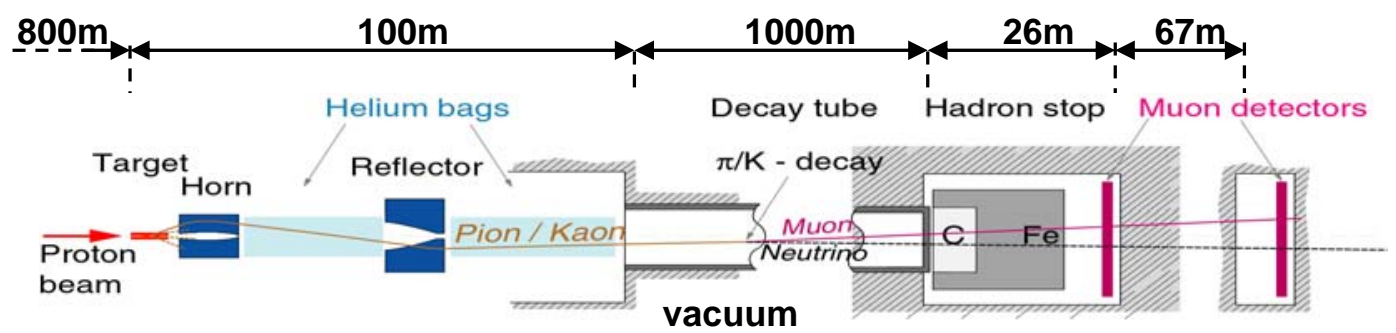

Figure 1: Layout of a typical conventional neutrino beam line (this example: CNGS).

In the following, the more recent and the presently operating neutrino beam facilities are described. Since most facilities had only minor difficulties with the proton beam operation, the emphasis here is on the target and secondary beam elements. Results and main lessons learnt are summarized. The T2K facility presently under construction in Japan is also mentioned in this context. 


\section{2. $\mathbf{K} 2 \mathbf{K}$}

$\mathrm{K} 2 \mathrm{~K}$ was the first long baseline neutrino oscillation experiment, aiming to measure oscillation parameters indicated by atmospheric neutrino experiment. The data taking period lasted from March 1999 until November 2004.

Neutrinos were produced using a $12 \mathrm{GeV}$ proton beam from KEK-PS with a cycle length of $2.2 \mathrm{sec}$ and a beam spill of $1.1 \mathrm{~ms}$ with $6 \cdot 10^{12}$ protons/spill. The average neutrino energy was about $1.3 \mathrm{GeV}$. An energy dependent deficit of muon neutrinos was observed in the 'far detector' Super-Kamiokande, $250 \mathrm{~km}$ distant from the neutrino production.

\subsection{K2K Secondary Neutrino Beam Line}

The $\mathrm{K} 2 \mathrm{~K}$ aluminum target was $66 \mathrm{~cm}$ long, with a diameter of $30 \mathrm{~mm}$ (initially $20 \mathrm{~mm}$ ) and was an integral part of the inner conductor in the first horn. There were two water cooled horns supplied with a current of $250 \mathrm{kA}$ and a pulse length of $2.5 \mathrm{~ms}$. The $200 \mathrm{~m}$ long decay volume started $19 \mathrm{~m}$ downstream of the target and it was filled with helium at 1atm. The hadron absorber at the end of the decay tube consisted of $3.5 \mathrm{~m}$ iron and $2 \mathrm{~m}$ concrete. Muon profile and intensity were measured using ionization chambers and silicon pad detectors installed downstream of the beam dump.

In order to measure the direction, flux and energy spectrum of the neutrinos at KEK a 'near detector' was installed $294 \mathrm{~m}$ downstream the target. This detector consisted of two systems: a 1 kton water Cherenkov detector and a fine-grained detector (muon range detector, scintillating fiber and bar trackers).

\subsection{Results and Lessons from the K2K Run}

During the data taking period between 1999 and 2004 in total $1.049 \cdot 10^{20}$ protons were delivered out of which $0.922 \cdot 10^{20}$ protons were used for the analysis. 112 neutrino events were observed in Super-K, while the expected number of events was 158.1+9.2/-8.6 in case of no oscillation. This results into $\Delta \mathrm{m}_{23}^{2}=(2.8 \pm 0.4) \cdot 10^{-3} \mathrm{eV}^{2}$ at $\sin ^{2}\left(2 \theta_{23}\right)=1(90 \% \mathrm{CL})$ [2].

The K2K horns were designed for $10^{7}$ cycles. Every year the horns were preventively exchanged. There was no facility for remote handling of the horns. The first horn-1 had to be exchanged very early in the run (July 1999); It was found that the $20 \mathrm{~mm}$ diameter Aluminum target rod (original design) was broken. After in-depth analysis, it was decided that the Aluminum target must have a diameter of $30 \mathrm{~mm}$. In total five horn-1 and four horn-2 were used. In November 2004 the inner conductor of horn-1 broke. At this point, the radiation levels were too high for a horn replacement. Since the delivered protons on target had meanwhile reached the design value of $10^{20}$, the K2K run was terminated in December 2004.

The $\mathrm{K} 2 \mathrm{~K}$ experience seems to indicate that the target should be decoupled from the inner conductor of the horn, in order to avoid fatigue caused from heating and vibrations. It also shows the importance of remote handling of the equipment installed in the target cavern as high radiation levels are already reached after some months of operation. 


\section{MiniBooNE}

MiniBooNE tests the LSND indication, i.e. an excess of electron neutrino candidates at the $\Delta \mathrm{m}^{2} \sim 1 \mathrm{eV}^{2}$ scale. Keeping the same experimental constraints for $\mathrm{L} / \mathrm{E}(500 \mathrm{MeV} / 500 \mathrm{~m})$ but having different signal signatures, backgrounds and sources of systematic errors, MiniBooNE searches for $v_{\mu} \rightarrow v_{\mathrm{e}}$ oscillation in an 'appearance experiment'.

The muon-neutrinos are produced by the $8 \mathrm{GeV}$ Fermilab Booster. The proton beam has a maximal intensity of $5 \cdot 10^{12}$ protons per $1.6 \mu$ s beam spill at a rate of $5 \mathrm{~Hz}$. The neutrino detector of $12.2 \mathrm{~m}$ diameter is situated $541 \mathrm{~m}$ downstream the target. It is filled with $800 \mathrm{t}$ pure mineral oil and lined with photomultiplier tubes (PMTs).

\subsection{MiniBooNE Secondary Neutrino Beam Line}

The $71 \mathrm{~cm}$ long MiniBooNE target, made of Beryllium, is segmented into seven 'slugs'. It is cantilevered into the neck of the horn. The target is air-cooled. The (single) horn is powered with $170 \mathrm{kA}$ with a pulse length of $140 \mu \mathrm{s}$. It is designed for $10^{8}$ cycles. A spare horn is available. The polarity of the horn can be changed within 1-2 weeks. The charged pions and kaons pass through a $60 \mathrm{~cm}$ diameter collimator and decay in the $50 \mathrm{~m}$ long decay tunnel. The tunnel has $1.8 \mathrm{~m}$ diameter and is filled with air at 1 bar. In addition to the hadron absorber downstream of the decay tunnel there is also a moveable iron absorber at $25 \mathrm{~m}$ (halfway). This absorber can be lowered into the tunnel to provide systematic checks on the electron-neutrino contamination from the muon decays. Both absorbers contain muon monitors (ionization chambers).

\subsection{Results and Lessons from the MiniBooNE Run}

Two independent blind analyses have been carried out with the result that no evidence for $v_{\mu} \rightarrow v_{e}$ oscillation has been observed [3]. Since the start of the experiment in 2002 until now (10 Aug 08) in total $1.17 \cdot 10^{21}$ protons on target have been accumulated. This corresponds to nearly 900000 neutrino events. Currently MiniBooNE runs in anti-neutrino mode, in order to search for anti-neutrino disappearance as well as to continue anti-neutrino cross-section measurements.

In 2003 , after $\sim 10^{8}$ pulses, the horn had to be exchanged due to a water leak and a ground fault caused most probable by galvanic corrosion at a bellows seal in a small volume of trapped water. The re-designed new horn is still running and has now collected $\sim 2 \cdot 10^{8}$ cycles. MiniBooNE provides a demonstration that mechanical fatigue may not be the major issue for horn lifetime: In a humid and radioactive environment much care should be taken in the design of the auxiliary systems of the horns as well as in the choice of materials.

During the early anti-neutrino run in 2006 a drop in the event yield has been observed. The reason was that the hardened steel chains of several absorber plates from the $25 \mathrm{~m}$ movable absorber were weakened by the radioactive atmosphere in the decay tunnel and, consequently, some absorber plates fell into the beam line. The plates were remounted using softer steel which is not subject to the hydrogen embrittlement effect. One may conclude that, in order to reduce aggressive radicals in the decay tube, air inside the tube should be avoided. Note that the decay 
tube of K2K was filled with helium, and T2K has chosen the same option. In December 2007, NuMI has switched from vacuum to a helium-filled decay tube. The decay tube in CNGS is under vacuum.

\section{NuMI}

NuMI, Neutrinos at the Main Injector, also verifies the $v_{\mu} \rightarrow v_{\tau}$ mixing hypothesis through muon neutrino disappearance and makes precise measurements of the oscillation parameters $\Delta \mathrm{m}_{23}^{2}$ and $\sin ^{2} 2 \theta_{23}$. NuMI was commissioned in 2004 and is fully operational since 2005 . The disappearance of muon neutrinos is measured in the MINOS detector, a 5.4kton magnetized tracking detector in Minnesota, at $735 \mathrm{~km}$ from Fermilab.

The neutrinos are produced by the $120 \mathrm{GeV} / \mathrm{c}$ proton beam from the Main Injector at Fermilab. The average intensity per pulse in $2007 / 2008$ is $3 \cdot 10^{13}$ protons per $10 \mu$ s and a cycle length of $1.9 \mathrm{~s}$. The majority of the data are taken with the low energy version of the neutrino beam at $\sim 4 \mathrm{GeV}$.

\subsection{NuMI Secondary Neutrino Beam Line}

The NuMI target is made of 47 graphite segments, each $20 \mathrm{~mm}$ long and a cross-section of $6.4 \times 15 \mathrm{~mm}^{2}$. The target is water cooled and mounted on a rail-drive system with $2.5 \mathrm{~m}$ travel inside the horn. This allows changing remotely the neutrino energy spectrum. The two NuMI horns are water cooled, pulsed with $2 \mathrm{~ms}$ half-sine wave pulse of up to $200 \mathrm{kA}$ and are designed for $10^{7}$ cycles. Target and horns are hanging on support modules, suspended from the shielding modules and services are connected at the top of the shielding. Remote connection for water and current, as well as remote exchange is foreseen.

The decay tube of $2 \mathrm{~m}$ diameter is $675 \mathrm{~m}$ long and was under vacuum. Since December 2007 the tube is filled with Helium at 1 bar. The hadron absorber is made of $2.4 \mathrm{~m}$ Aluminum and $2.3 \mathrm{~m}$ iron. A hadron monitor upstream and three muon monitors downstream of the absorber are used to measure the spatial distribution of the charged hadrons and muons as well as to check the alignment of the beam.

The $1 \mathrm{kton}$ near detector, $1 \mathrm{~km}$ from the target, is functionally identical to the far detector and shares the basic detector technology and granularity.

\subsection{Results and Lessons from the NuMI Run}

The total number of protons on target so far is $\sim 5 \cdot 10^{20}$. Recent results for the oscillation parameters are $\Delta \mathrm{m}_{23}^{2}=(2.43 \pm 0.13) \cdot 10^{-3} \mathrm{eV}^{2}$ at $\sin ^{2} 2 \theta_{23}=1_{-0.05}$ [4].

Since the start-up of the NuMI run, the target and the horns faced several problems; target drive failure, horn ground fault, water leaks, water line contamination by resin beads, etc... Although the equipment design foresees complete replacement of radioactive elements, many of the problems occurring up to now were repairable. This is possible thanks to a work cell which is installed in the downstream part of the target area; the target or horns can be remotely moved into the shielded work cell onto a remote lifting table and can be investigated through lead-glass windows before and during repair work. Connections are done through the module on top (see 
Fig. 2). Hence during the design of secondary beam line equipment repair work must be kept in mind. Thorough tests before first beam should be performed. Proper tooling as well as training must be foreseen.
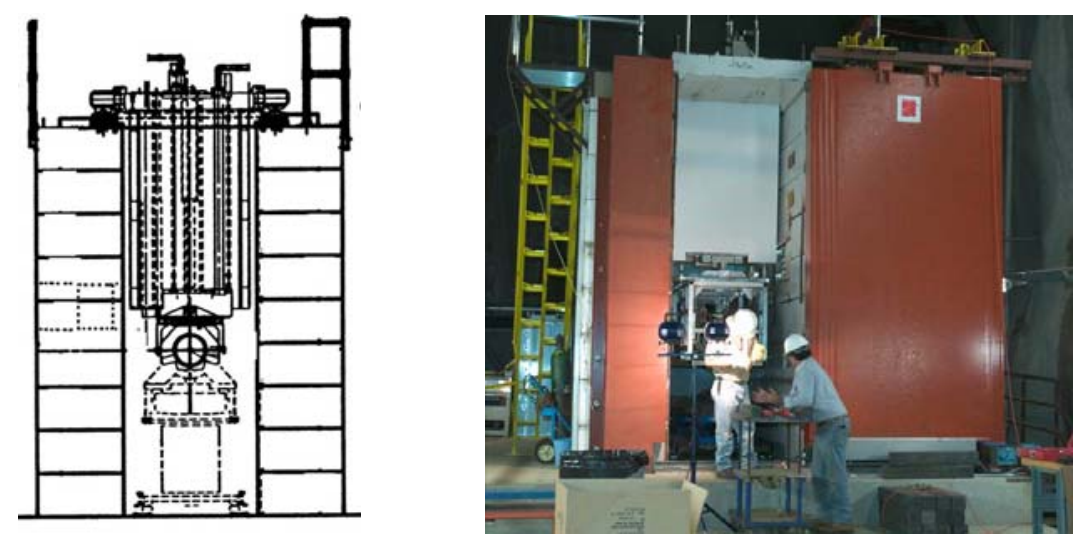

Figure 2: Sketch (left) and photo (right) of the NuMI Work cell.

A major issue at NuMI was the tritium levels in the water pumped from the NuMI tunnels. They were considerably higher than expected, although still very low compared to the regulatory limits. The source was traced to the tritium production in the steel surrounding the target chase. This was then carried to the tunnel water by moisture in the chase air. An efficient remedy was found using dehumidification of the target hall and the target chase air.

\section{CNGS}

CNGS, CERN Neutrinos to Gran Sasso, sends muon neutrinos with an average energy of $\sim 17 \mathrm{GeV} / \mathrm{c}$ from CERN over $732 \mathrm{~km}$ to Gran Sasso, Italy. There, two detectors are located: OPERA, a 1200t detector made of 146000 emulsion bricks and ICARUS, a 600t liquid Argon detector. CNGS is the first experiment in which the measurement of the oscillation parameters is done by observation of tau-neutrino appearance.

The muon neutrinos are produced from the $400 \mathrm{GeV} / \mathrm{c}$ SPS proton beam. The nominal intensity is $2.4 \cdot 10^{13}$ protons on target per $10.5 \mu$ s extraction. During the 6 s cycle, there are two extractions separated by $50 \mathrm{~ms}$.

CNGS was commissioned in 2006. During 2007 CNGS was running for 6 weeks [5]. The CNGS run 2008 started in June after the completion of the OPERA detector and finishing modifications in the CNGS facility. In 2008 so far (status 25 September) $0.9 \cdot 10^{19}$ protons on target have been gathered and OPERA has collected more than 730 brick events.

\subsection{CNGS Secondary Neutrino Beam Line}

The CNGS target unit is made of 13 graphite rods of $4 \mathrm{~mm}$ diameter each (first two rods have $5 \mathrm{~mm}$ diameter). The rods are $10 \mathrm{~cm}$ long and interspaced with $9 \mathrm{~cm}$. Five units are assembled into a target magazine, where one unit is used and the other four are kept as in-situ spares. The two magnetic horns are water cooled and pulsed twice every $6 \mathrm{~s}$ cycle with a current 
of 150kA (180kA) during a few milliseconds. Polarity change can be done remotely. Special features are implemented to exchange the target or the horns remotely.

The CNGS decay tube is $1000 \mathrm{~m}$ long, with a diameter of $2.45 \mathrm{~m}$ and is under vacuum at less than $1 \mathrm{mbar}$. Downstream of this tunnel, the hadron absorber, made of $3 \mathrm{~m}$ graphite and $15 \mathrm{~m}$ iron, is designed to absorb up to $100 \mathrm{~kW}$ of protons and hadrons. Two muon monitor stations, separated by $67 \mathrm{~m}$ of rock provide on-line feedback for the quality control of the neutrino beam. They are arranged in a cross-shaped array (see Fig. 3a) and measure the muon intensity and the vertical and horizontal muon profiles.

\subsection{Results and Lessons from the CNGS Run}

In the horizontal muon profiles an asymmetry between operating in neutrino mode (focusing of mesons with positive charge) and anti-neutrino mode (mesons with negative charge) has been observed. This can be explained due to the earth magnetic field in the $1000 \mathrm{~m}$ long decay tube which results in the profile shifts of the observed magnitude (see Fig. 3b). Due to the possibility of changing remotely the polarity this effect could be studied in detail.
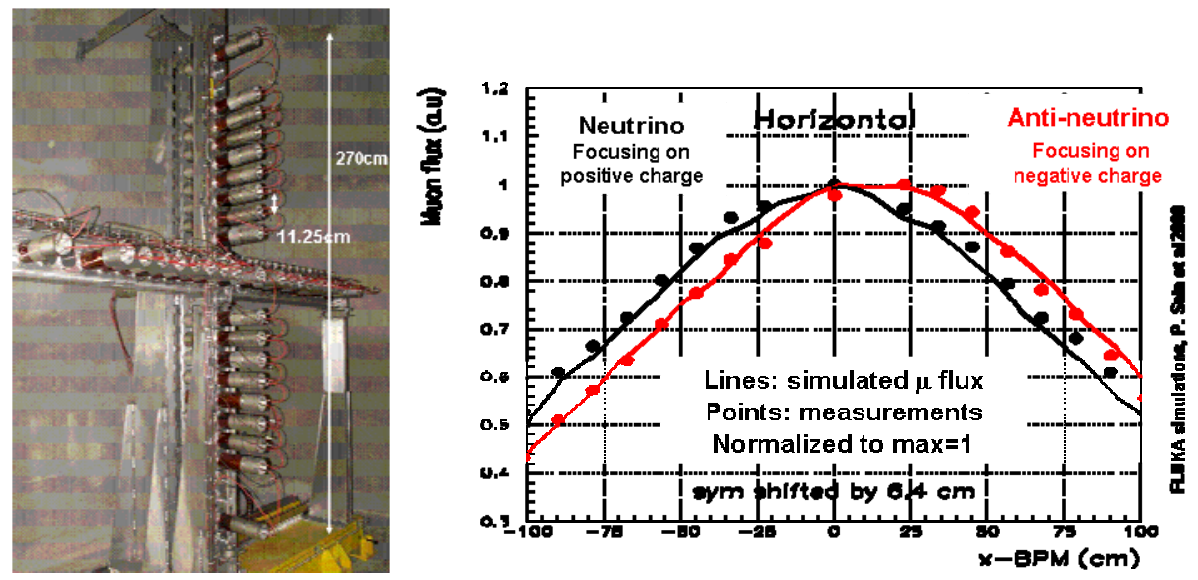

Figure 3: Left: CNGS Muon detector station. Right: measurements and simulations of the horizontal muon profiles in neutrino and anti-neutrino mode.

During the 2006 CNGS run, after only $4 \cdot 10^{5}$ horn pulses, a leak in a water outlet of the $2^{\text {nd }}$ horn cooling circuit has been detected. This was caused by a design fault in a ceramic insulator brazing. Thanks to detailed dose planning, tooling and training as well as additional local shielding a repair was possible.

CNGS has no surface building above the target and hence a large fraction of electronics is installed near the CNGS target area. During the CNGS run in 2007, a failure in the control of the ventilation system was caused by radiation effects (SEU, single event upsets due to high energy hadron fluence). The remedy was (a) to move as much electronics as possible out of the CNGS tunnel area and (b) to create in the tunnel a radiation safe area for the electronics by adding shielding. This decreased the radiation to electronics by up to a factor $10^{6}$. The lesson learnt: In these harsh environments the radiation hardness of the installed electronics and materials must be addressed already during the design phase. Standard electronics components should be avoided in these areas. 


\section{T2K}

T2K (Tokai to Kamioka) is an off-axis $v_{\mu} \rightarrow v_{\mathrm{e}}$ appearance experiments to measure the mixing angle $\theta_{13}$ as well as to perform precise measurements of $\Delta \mathrm{m}_{23}^{2}$ and $\sin ^{2} 2 \theta_{23}$. The low energy off-axis beam is tunable by changing the off-axis angle between $2^{\circ}$ and $2.5^{\circ}$ which corresponds to a neutrino energy $\mathrm{E}_{v}$ between $0.8-0.65 \mathrm{GeV}$.

First beam is expected in April 2009 [6]. The neutrinos will be produced at J-PARC from the $0.75 \mathrm{MW} 50 \mathrm{GeV}$ proton beam from the Main Ring MR and will be sent $295 \mathrm{~km}$ along to the 50kton Water Cherenkov Super-Kamiokande detector.

The $\mathrm{T} 2 \mathrm{~K}$ secondary beam design has profited a lot from the experiences gained in the current neutrino beam line: careful design and extensive testing of the equipment and all the accessory systems has been performed. The entire volume of the target station is filled with Helium in order to reduce the production of aggressive chemical substances and tritium. Remote handling of all equipment is foreseen.

\section{Summary}

The operation of high power neutrino beams at the present levels of up to $500 \mathrm{~kW}$ has proven challenging, in particular concerning the equipment downstream of the target. Lessons learnt, of which some have been outlined here, indicate critical issues to be considered in the preparation of future facilities in the multi-MW domain.

\section{Acknowledgement}

Many colleagues have provided information and comments concerning the operation of neutrino beam facilities. The contributions from Sam Childress, Ilias Efthymiopoulos, Konrad Elsener, Peter Kasper, Takashi Kobayashi, Sacha Kopp, Malika Meddahi, Ans Pardons, Kazuhiro Tanaka and Heinz Vincke are particularly acknowledged.

\section{References}

[1] A. Pardons, Horn Operational Experience in K2K, MiniBooNE, NuMI and CNGS, in proceedings of $10^{\text {th }}$ International Workshop on Neutrino Factories, Super beams and Beta beams (Nufact08), June 30 - July 5 2008, Valencia, Spain PoS(NUFACT08)096

[2] Phys.Rev.D74:072003, 2006

[3] Phys.Rev.Lett.98, 231801, 2007

[4] Phys.Rev.Lett.arXiv:0806.2237, 2008

[5] E. Gschwendtner et al, First Operational Experience with the CNGS Facility, Nufact07, 6-11 August 2007, Okayama, Japan, AIP Conf. Proc.: 981 (2008) pp 23-25, 2008

[6] T. Ishida, Neutrino Facility at J-PARC, Workshop on Neutrinos and Beams, 5-7 May 2008, Darjeeling, India, 2008 